Proyecciones Journal of Mathematics Vol. 36, No 1, pp. 29-44, March 2017. Universidad Católica del Norte Antofagasta - Chile

\title{
On the hyperstability of a quartic functional equation in Banach spaces
}

\author{
Nordine Bounader \\ University of IBN Tofail, Morocco \\ Received: August 2016. Accepted : October 2016
}

\begin{abstract}
In this paper, we establish some hyperstability results of the following functional equation

$$
f(2 x+y)+f(2 x-y)=4(f(x+y)+f(x-y))+24 f(x)-6 f(y)
$$

in Banach spaces.
\end{abstract}

Subjclass [2010] : Primary 39B82, 39B62; Secondary 47H14, 47H10.

Keywords : Hyperstability, Quartic functional equation, fixed point theorem. 


\section{Introduction and Preliminaries}

The stability problem of functional equations was posed for the first time by Ulam [23] in the year 1940. Ulam stated the problem as follows:

Let $(G,$.$) be a group and let (H, ., d)$ be a metric group with the metric $d$. Given $\delta>0$, does there exist $\epsilon>0$ such that if a mapping $h: G \rightarrow H$ satisfies the inequality

$$
d(h(x y), h(x) h(y)) \leq \delta
$$

for all $x, y \in G$, then there is a homomorphism $a: G \rightarrow H$ with

$$
d(h(x), a(x)) \leq \epsilon
$$

for all $x \in G$

Ulam's problem was partially solved by Hyers in 1941 in the context of Banach spaces with $\epsilon=\delta$ as shown below [15].

Theorem 1.1 (D. H. Hyers (1941)). Let $E$ be a normed vector space, $F$ a Banach space and suppose that the mapping $f: E \rightarrow F$ satisfies the inequality

$$
\|f(x+y)-f(x)-f(y)\| \leq \epsilon
$$

for all $x, y \in E$ where $\epsilon$ is a constant. Then the limit

$$
T(x)=\lim _{n \rightarrow \infty} 2^{-n} f\left(2^{n} x\right)
$$

exists for each $x \in E$ and $T$ is the unique additive mapping satisfying

$$
\|f(x)-T(x)\| \leq \epsilon
$$

for all $x \in E$. Also, if for each $x$ the function $t \rightarrow f(t x)$ from $\mathbf{R}$ to $F$ is continuous for each fixed $x$, then $T$ is linear. If $f$ is continuous at a single point of $E$, then $T$ is continuous in $E$.

Aoki [1], Z. Gajda [13] and Th.M. Rassias [19] provided a generalization of the Hyers theorem for additive and linear mappings, respectively, by allowing the Cauchy difference to be unbounded.

Theorem 1.2. Let $f: E \rightarrow F$ be a mapping from a real normed vector space $E$ into a Banach space $F$ satisfying the inequality

$$
\|f(x+y)-f(x)-f(y)\| \leq \theta\left(\|x\|^{p}+\|y\|^{p}\right),
$$


for all $x, y \in E \backslash\{0\}$, where $\theta$ and $p$ are constants with $\theta>0$ and $p \neq 1$. Then there exists a unique additive mapping $T: E \rightarrow F$ such that

$$
\|f(x)-T(x)\| \leq \frac{\theta}{\left|1-2^{p-1}\right|}\|x\|^{p}, \quad \forall x \in E \backslash\{0\} .
$$

Theorem 1.2 is due to Aoki [1] for $0<p<1$ (see also [19]); Gajda [13] for $p>1$; Hyers [15] for $p=0$ and Th. M. Rassias [20] for $p<0$ (see [[21], page 326] and [5]). Recently, for $p<0$ a stronger result is valid, that is hyperstability; information concerning it can be found in [9].

J. M. Rassias [22](in1999) introduced the quartic functional equation $(1.3) f(x+2 y)-4 f(x+y)+6 f(x)-4 f(x-y)+f(x-2 y)=24 f(y)$

and investigated stability properties of the quartic functional equation (1.3). Since $f(x)=x^{4}$ satisfies the functional equation (1.3), the function is called quartic function equation.

S. H. Lee, S. M. Im and I. S. Shwang [16] determined the general solution of the quartic functional equation

$$
(1.4) f(2 x+y)+f(2 x-y)=4(f(x+y)+f(x-y))+24 f(x)-6 f(x)
$$

We say a functional equation $D$ is hyperstable if any function $f$ satisfying the equation $D$ approximately is a true solution of $D$. The term hyperstability was used for first time in [17]. However, it seems that the first hyperstability result was published in [4] and concerned the ring homomorphisms. The hyperstability results for Cauchy equation were investigated by Brzdek in $[8,10,11]$. Gselmann in [14] studied the hyperstability of the parametric fundamental equation of information. In [3] Bahyrycz and Piszczek provided the hyperstability of the Jensen functional equation.

In this paper, we present the hyperstability results for the quartic functional equation (1.4) in Banach spaces.

The method of the proof of the main results is motivated by an idea used by Brzdek in $[8,10,11]$ and further by Piszczek in [18]. It is based on a fixed point theorem for functional spaces obtained by Brzdek et al. (see [[7], Theorem 1]). 
Throughout the paper, $\mathbf{N}, \mathbf{R}, \mathbf{R}_{+}$and $\mathbf{N}_{m_{0}}$ denote the set of all positive integers, the set of real numbers, the set of positive real numbers and the set of all integers greater than or equal to $m_{0}$, respectively.

First, we take the following three hypotheses (all notations come from [7]).

(H1) $X$ is a nonempty set, $Y$ is a Banach space, $f_{1}, \ldots . f_{k}: X \rightarrow X$ and $L_{1}, \ldots . L_{k}: X \rightarrow \mathbf{R}_{+}$are given.

(H2) $\mathcal{T}: Y^{X} \rightarrow Y^{X}$ is an operator satisfying the inequality

$$
\|\mathcal{T} \xi(x)-\mathcal{T} \mu(x)\| \leq \sum_{i=1}^{k} L_{i}(x)\left\|\xi\left(f_{i}(x)\right)-\mu\left(f_{i}(x)\right)\right\|
$$

for all $\xi, \mu \in Y^{X}, x \in X$.

(H3) $\Lambda: \mathbf{R}_{+}{ }^{X} \rightarrow \mathbf{R}_{+}{ }^{X}$ is a linear operator defined by

$$
\Lambda \delta(x):=\sum_{i=1}^{k} L_{i}(x) \delta\left(f_{i}(x)\right)
$$

for all $\delta \in \mathbf{R}_{+}{ }^{X}, x \in X$.

The mentioned fixed point theorem is stated as follows.

Theorem 1.3. Let hypotheses (H1)-(H3) be valid and functions $\varepsilon: X \rightarrow$ $\mathbf{R}_{+}$and let $\varphi: X \rightarrow Y$ fulfil the following two conditions:

$$
\begin{gathered}
\|\mathcal{T} \varphi(x)-\varphi(x)\| \leq \varepsilon(x), \quad x \in X \\
\varepsilon^{*}(x):=\sum_{n=0}^{\infty} \Lambda^{n} \varepsilon(x)<\infty, \quad x \in X .
\end{gathered}
$$

Then, there exists a unique fixed point $\psi$ of $\mathcal{T}$ with

$$
\|\varphi(x)-\psi(x)\| \leq \varepsilon^{*}(x), \quad x \in X
$$

Moreover

$$
\psi(x)=\lim _{n \rightarrow \infty} \mathcal{T}^{n} \varphi(x), \quad x \in X .
$$

For more information on the issue of hyperstability of functional equation we refer to $[6]$ 


\section{Hyperstability Results of eq (1.4)}

The following theorems and corollaries are the main results in this paper and concern the hyperstability of the functional equation (1.4). Moreover the results that we will obtained in these theorems corresponds the results obtained in $[3,11]$.

Theorem 2.1. Let $X$ be a normed space, $Y$ be a Banach space, $c \geq 0$ and $p, q, \in \mathbf{R}$ such that $p+q<0$. If $f: X \rightarrow Y$ satisfies

$$
\|f(2 x+y)+f(2 x-y)-[4(f(x+y)+f(x-y))+24 f(x)-6 f(y)]\|
$$

$$
\leq c\|x\|^{p}\|y\|^{q}
$$

for all $x, y \in X \backslash\{0\}$ such that $x+y \neq 0, x-y \neq 0,2 x+y \neq 0,2 x-y \neq 0$.

Then $f$ is a solution of (1.4) on $X \backslash\{0\}$.

Proof. We consider only the case $q<0$, the case $p<0$ is analogous. Replacing $y$ by $m x$ where $m \in \mathbf{N}_{3}$ in (2.1), we obtain that

$\|24 f(x)-[6 f(m x)-4 f((m+1) x)-4 f((1-m) x)+f((2+m) x)+f((2-m) x)]\|$

$$
\leq c m^{q}\|x\|^{p+q}
$$

for all $x \in X \backslash\{0\}$.

This inequality is equivalent to the following

$$
\begin{aligned}
\| f(x)-\left[\frac{1}{4} f(m x)-\frac{1}{6} f((m+1)\right. & \left.x)-\frac{1}{6} f((1-m) x)+\frac{1}{24} f((2+m) x)+\frac{1}{24} f((2-m) x)\right] \| \\
\leq & \frac{1}{24} c m^{q}\|x\|^{p+q}
\end{aligned}
$$

for all $x \in X \backslash\{0\}$.

Further put

$$
\begin{gathered}
\mathcal{T} \xi(x):=\frac{1}{4} \xi(m x)-\frac{1}{6} \xi((m+1) x)-\frac{1}{6} \xi((1-m) x)+\frac{1}{24} \xi((2+m) x)+\frac{1}{24} \xi((2-m) x), \\
x \in X \backslash\{0\}, \xi \in Y^{X \backslash\{0\}},
\end{gathered}
$$




$$
\varepsilon(x):=\frac{1}{24} c m^{q}\|x\|^{p+q}, \quad x \in X \backslash\{0\} .
$$

Then the inequality (2.2) takes the form

$$
\|\mathcal{T} f(x)-f(x)\| \leq \varepsilon(x), \quad x \in X \backslash\{0\} .
$$

The operator

$$
\begin{gathered}
\Lambda \delta(x):=\frac{1}{4} \delta(m x)+\frac{1}{6} \delta((m+1) x)+\frac{1}{6} \delta((1-m) x)+\frac{1}{24} \delta((2+m) x)+\frac{1}{24} \delta((2-m) x), \\
x \in X \backslash\{0\}, \delta \in \mathbf{R}_{+} X \backslash\{0\}
\end{gathered}
$$

has the form described in (H3) with $k=5$ and

$f_{1}(x)=m x, f_{2}(x)=(m+1), f_{3}(x)=(1-m) x, f_{4}(x)=(2+m) x, f_{5}(x)=(2-m) x$

and

$$
L_{1}(x)=\frac{1}{4}, L_{2}(x)=\frac{1}{6}, L_{3}(x)=\frac{1}{6}, L_{4}(x)=\frac{1}{24}, L_{5}(x)=\frac{1}{24}, \forall x \in X \backslash\{0\} .
$$

Moreover, for every $\xi, \mu \in Y^{X \backslash\{0\}}$ and $x \in X \backslash\{0\}$, we obtain $\|T \xi(x)-T \mu(x)\|$

$=\| \frac{1}{4}(\xi-\mu)(m x)-\frac{1}{6}(\xi-\mu)((m+1) x)-\frac{1}{6}(\xi-\mu)((1-m) x)+$

$\frac{1}{24}(\xi-\mu)((2+m) x)+\frac{1}{24}(\xi-\mu)((2-m) x) \|$

$\leq \frac{1}{4}\left\|(\xi-\mu) f_{1}(x)\right\|+\frac{1}{6}\left\|(\xi-\mu) f_{2}(x)\right\|+\frac{1}{6}\left\|(\xi-\mu) f_{3}(x)\right\|+$

$\frac{1}{24}\left\|(\xi-\mu) f_{4}(x)\right\|+\frac{1}{24}\left\|(\xi-\mu) f_{5}(x)\right\|$

$\leq \sum_{i=1}^{5} L_{i}(x)\left\|\xi\left(f_{i}(x)\right)-\mu\left(f_{i}(x)\right)\right\|$.

So, (H2) is valid. Next, we can find $m_{0} \in \mathbf{N}_{3}$ such that

$$
\begin{gathered}
\alpha_{m}:=\frac{1}{4} m^{p+q}+\frac{1}{6}(m+1)^{p+q}+\frac{1}{6}|1-m|^{p+q}+\frac{1}{24}(2+m)^{p+q}+\frac{1}{24}|2-m|^{p+q}<1 \\
\text { for all } m \geq m_{0} .
\end{gathered}
$$

Therefore, we obtain that 
On the hyperstability of a quartic functional equation in Banach ... 35

$\varepsilon^{*}(x):=\sum_{n=0}^{\infty} \Lambda^{n} \varepsilon(x)=\frac{1}{24} c m^{q}\|x\|^{p+q} \sum_{n=0}^{\infty} \alpha_{m}{ }^{n}=\frac{c m^{q}\|x\|^{p+q}}{24\left(1-\alpha_{m}\right)}<\infty$, $\forall x \in X \backslash\{0\} ; \quad \forall m \geq m_{0}$.

Thus according to Theorem 1.3, for each $m \geq m_{0}$ there exists a unique solution $F_{m}: X \backslash\{0\} \rightarrow Y$ of the equation

$$
\begin{gathered}
F_{m}(x)=\frac{1}{4} F_{m}(m x)-\frac{1}{6} F_{m}(( \\
(m+1) x)-\frac{1}{6} F_{m}((1-m) x)+\frac{1}{24} F_{m}((2+m) x) \\
+\frac{1}{24} F_{m}((2-m) x)
\end{gathered}
$$

for all $x \in X \backslash\{0\}$ such that

$$
\left\|f(x)-F_{m}(x)\right\| \leq \varepsilon_{m}^{*}(x), \quad x \in X \backslash\{0\}, \quad m \geq m_{0} .
$$

Moreover $F_{m}(x)=\lim _{n \rightarrow \infty} T^{n} F_{m}(x), x \in X \backslash\{0\}$.

To prove that $F_{m}$ satisfying (1.4) on $X \backslash\{0\}$, observe that

$\left\|\mathcal{T}^{n} f(x)-\left[\frac{1}{4} \mathcal{T}^{n} f(y)-\frac{1}{6} \mathcal{T}^{n} f(x+y)-\frac{1}{6} \mathcal{T}^{n} f(x-y)+\frac{1}{24} \mathcal{T}^{n} f(2 x+y)+\frac{1}{24} \mathcal{T}^{n} f(2 x-y)\right]\right\|$

$$
\leq \frac{1}{24} c\|x\|^{p}\|y\|^{q} \alpha_{m}^{n}
$$

for all $x, y \in X \backslash\{0\}$ such that $x+y \neq 0, x-y \neq 0,2 x+y \neq 0,2 x-y \neq 0$, and $n \in \mathbf{N}$.

Indeed, if $n=0$, then (2.3) is simply (2.1). So, fix $n \in \mathbf{N}$ and suppose that (2.3) holds for $n$ and all $x, y \in X \backslash\{0\}$ such that $x+y \neq 0, x-y \neq$ $0,2 x+y \neq 0,2 x-y \neq 0$. Then 


$$
\begin{aligned}
& \| T^{n+1} f(x)-\left[\frac{1}{4} T^{n++1} f(y)-\frac{1}{6} T^{n+1} f(x+y)-\frac{1}{6} T^{n+1} f(x-y)\right. \\
& \left.+\frac{1}{24} T^{n+1} f(2 x+y)+\frac{1}{24} T^{n+1} f(2 x-y)\right] \| \\
& =\| \frac{1}{4} T^{n} f(m x)-\frac{1}{6} T^{n} f((m+1) x)-\frac{1}{6} T^{n} f((1-m) x) \\
& +\frac{1}{24} T^{n} f((2+m) x)+\frac{1}{24} T^{n} f((2-m) x) \\
& -\frac{1}{4}\left[\frac{1}{4} T^{n} f(m y)-\frac{1}{6} T^{n} f((m+1) y)-\frac{1}{6} T^{n} f((1-m) y)\right. \\
& \left.+\frac{1}{24} T^{n} f((2+m) y)+\frac{1}{24} T^{n} f((2-m) y)\right] \\
& +\frac{1}{6}\left[\frac{1}{4} T^{n} f(m(x+y))-\frac{1}{6} T^{n} f((m+1)(x+y))\right. \\
& -\frac{1}{6} T^{n} f((1-m)(x+y))+\frac{1}{24} T^{n} f((2+m)(x+y))+ \\
& \left.\frac{1}{24} T^{n} f((2-m)(x+y))\right] \\
& +\frac{1}{6}\left[\frac{1}{4} T^{n} f(m(x-y))-\frac{1}{6} T^{n} f((m+1)(x-y))\right. \\
& -\frac{1}{6} T^{n} f((1-m)(x-y))+\frac{1}{24} T^{n} f((2+m)(x-y))+ \\
& \left.\frac{1}{24} T^{n} f((2-m)(x-y))\right] \\
& -\frac{1}{24}\left[\frac{1}{4} T^{n} f(m(2 x+y))-\frac{1}{6} T^{n} f((m+1)(2 x+y))\right. \\
& -\frac{1}{6} T^{n} f((1-m)(2 x+y))+\frac{1}{24} T^{n} f((2+m)(2 x+y)) \\
& \left.+\frac{1}{24} T^{n} f((2-m)(2 x+y))\right]-\frac{1}{24}\left[\frac{1}{4} T^{n} f(m(2 x-y))-\frac{1}{6} T^{n} f((m+1)(2 x-y))\right. \\
& -\frac{1}{6} T^{n} f((1-m)(2 x-y))+\frac{1}{24} T^{n} f((2+m)(2 x-y)) \\
& \left.+\frac{1}{24} T^{n} f((2-m)(2 x+y))\right] \\
& =\| \frac{1}{4}\left[T^{n} f(m x)-\left[\frac{1}{4} T^{n} f(m y)-\frac{1}{6} T^{n} f(m(x+y))\right.\right. \\
& -\frac{1}{6} T^{n} f(m(x-y))+\frac{1}{24} T^{n} f(m(2 x+y))+ \\
& \left.\left.\frac{1}{24} T^{n} f(m(2 x-y))\right]\right] \\
& -\frac{1}{6}\left[T^{n} f((m+1) x)-\left[\frac{1}{4} T^{n} f((m+1) y)-\frac{1}{6} T^{n} f((m+1)(x+y))-\right.\right. \\
& \left.\left.\frac{1}{6} T^{n} f((m+1)(x-y))+\frac{1}{24} T^{n} f((m+1)(2 x+y))+\frac{1}{24} T^{n} f((m+1)(2 x-y))\right]\right] \\
& -\frac{1}{6}\left[T^{n} f((1-m) x)-\left[\frac{1}{4} T^{n} f((1-m) y)\right.\right. \\
& -\frac{1}{6} T^{n} f((1-m)(x+y))- \\
& \left.\left.\frac{1}{6} T^{n} f((1-m)(x-y))+\frac{1}{24} T^{n} f((1-m)(2 x+y))+\frac{1}{24} T^{n} f((1-m)(2 x-y))\right]\right] \\
& +\frac{1}{24}\left[T^{n} f((2+m) x)-\left[\frac{1}{4} T^{n} f((2+m) y)\right.\right.
\end{aligned}
$$


for all $x, y \in X \backslash\{0\}$ such that $x+y \neq 0, x-y \neq 0,2 x+y \neq 0,2 x-y \neq 0$.

Thus, by induction, we have shown that (2.3) holds for all $x, y, \in X \backslash\{0\}$ such that $x+y \neq 0, x-y \neq 0,2 x+y \neq 0,2 x-y \neq 0$, and for all $n \in \mathbf{N}$.

Letting $n \rightarrow \infty$ in (2.3), we obtain

$F_{m}(x)=\frac{1}{4} F_{m}(y)-\frac{1}{6} F_{m}(x+y)-\frac{1}{6} F_{m}(x-y)+\frac{1}{24} F_{m}(2 x+y)+\frac{1}{24} F_{m}(2 x-y)$

for all $x, y \in X \backslash\{0\}$ such that $x+y \neq 0, x-y \neq 0,2 x+y \neq 0,2 x-y \neq 0$,. So, we find a sequence $\left(F_{m}\right)_{m \geq m_{0}}$ satisfies (1.4) on $X \backslash\{0\}$ such that

$$
\left\|f(x)-F_{m}(x)\right\| \leq \frac{c m^{q}}{24\left(1-\alpha_{m}\right)}\|x\|^{p+q}, \forall x \in X-\{0\}, \forall m \geq m_{0} .
$$

It follows, with $m \rightarrow \infty$, that $f$ is a solution of (1.4) on $X \backslash\{0\}$.

Corollary 2.1.1. Let $X$ be a normed space and $Y$ be a Banach space. Let $H: X^{2} \rightarrow Y$ be a mapping such that $H\left(x_{0}, y_{0}\right) \neq 0$ for some $x_{0}, y_{0} \in X$ and

$$
\|H(x, y)\| \leq c\|x\|^{p}\|y\|^{q},
$$

for all $x, y \in X \backslash\{0\}$ such that $x+y \neq 0, x-y \neq 0,2 x+y \neq 0,2 x-y \neq 0$, where $c>0$ and $p, q \in \mathbf{R}$. Assume that the numbers $p, q$ satisfy $p+q<0$.

Then the functional equation

$H(2 x+y)+h(2 x-y)=H(x, y)+4(H(x+y)+H(x-y))+24 H(x)-6 H(y)$,

$$
x, y \in X \backslash\{0\}
$$

has no solution in the class of functions $H: X \rightarrow Y$.

Proof. Suppose that $H: X \rightarrow Y$ is a solution to (2.5). Then (2.1) holds, and consequently, according to the above theorem, $H$ is quartic function equation on $X \backslash\{0\}$, which means that $H\left(x_{0}, y_{0}\right)=0$. This is a contradiction.

In a similar way we can prove the following theorem.

Theorem 2.2. Let $X$ be a normed space, $Y$ be a Banach space, $c \geq 0$ and $p \in \mathbf{R}$ such that $p<0$. If $f: X \rightarrow Y$ satisfies

$$
\|f(2 x+y)+f(2 x-y)-[4(f(x+y)+f(x-y))+24 f(x)-6 f(y)]\|
$$

$$
\leq c\left(\|x\|^{p}+\|y\|^{p}\right)
$$

for all $x, y \in X \backslash\{0\}$ such that $x+y \neq 0, x-y \neq 0,2 x+y \neq 0,2 x-y \neq 0$. Then $f$ is a solution of (1.4) on $X \backslash\{0\}$. 
Proof. Replacing $x$ by $(1+m) x$ and $y$ by $m x$ where $m \in \mathbf{N}_{1}$ in (2.6). We obtain that

$\|4 f(x)-[f((3 m+2) x)+f((m+2) x)-4 f((1+2 m) x)-24 f((1+m) x)+6 f(m x)]\|$

$$
\leq c\left((1+m)^{p}+m^{p}\right)\|x\|^{p}
$$

for all $x \in X \backslash\{0\}$.

This inequality is equivalent to the following

$$
\begin{aligned}
\| f(x)-\left[\frac{1}{4} f((3 m+2) x)+\right. & \left.\frac{1}{4} f((m+2) x)-f((1+2 m) x)-6 f((1+m) x)+\frac{3}{2} f(m x)\right] \| \\
& \leq \frac{1}{4} c\left((1+m)^{p}+m^{p}\right)\|x\|^{p}
\end{aligned}
$$

for all $x \in X \backslash\{0\}$.

Further put

$$
\begin{gathered}
\mathcal{T} \xi(x):=\frac{1}{4} \xi((3 m+1) x)+\frac{1}{4} \xi((m+2) x)-\xi((1+2 m) x)-6 \xi((1+m) x)+\frac{3}{2} \xi(m x), \\
x \in X \backslash\{0\}, \xi \in Y^{X \backslash\{0\},} \\
\varepsilon(x):=\frac{1}{4} c\left((1+m)^{p}+m^{p}\right)\|x\|^{p}, \quad x \in X \backslash\{0\} .
\end{gathered}
$$

Then the inequality (2.7) takes the form

$$
\|\mathcal{T} f(x)-f(x)\| \leq \varepsilon(x), \quad x \in X \backslash\{0\} .
$$

The operator

$$
\begin{gathered}
\Lambda \delta(x):=\frac{1}{4} \delta((3+2 m) x)+\frac{1}{4} \delta((m+2) x)+\delta((1+2 m) x)+6 \delta((1+m) x)+\frac{3}{2} \delta(m x), \\
x \in X \backslash\{0\}, \delta \in \mathbf{R}_{+} X \backslash\{0\}
\end{gathered}
$$

has the form described in (H3) with $k=5$ and

$$
\begin{gathered}
f_{1}(x)=(3 m+2) x, f_{2}(x)=(m+2) x, f_{3}(x)=(1+2 m) x, f_{4}(x)=(1+m) x, \\
f_{5}(x)=m x
\end{gathered}
$$

and 
$L_{1}(x)=\frac{1}{4}, L_{2}(x)=\frac{1}{4}, L_{3}(x)=1, L_{4}(x)=6, L_{5}(x)=\frac{3}{2}, \forall x \in X \backslash\{0\}$.

Moreover, for every $\xi, \mu \in Y^{X \backslash\{0\}}$ and $x \in X \backslash\{0\}$, we obtain

$$
\begin{aligned}
& \|T \xi(x)-T \mu(x)\| \\
& =\| \frac{1}{4}(\xi-\mu)((3 m+1) x)+\frac{1}{4}(\xi-\mu)((m+2) x)-(\xi-\mu)((1+2 m) x)+ \\
& -6(\xi-\mu)((1+m) x)+\frac{3}{2}(\xi-\mu)(m x) \| \\
& \leq \frac{1}{4}\left\|(\xi-\mu) f_{1}(x)\right\|+\frac{1}{4}\left\|(\xi-\mu) f_{2}(x)\right\|+\left\|(\xi-\mu) f_{3}(x)\right\|+ \\
& 6\left\|(\xi-\mu) f_{4}(x)\right\|+\frac{3}{2}\left\|(\xi-\mu) f_{5}(x)\right\| \\
& \leq \sum_{i=1}^{5} L_{i}(x)\left\|\xi\left(f_{i}(x)\right)-\mu\left(f_{i}(x)\right)\right\|
\end{aligned}
$$

So, (H2) is valid. Next, we can find $m_{0} \in \mathbf{N}_{1}$ such that $\alpha_{m}:=\frac{1}{4}((3 m+1))^{p}+\frac{1}{4}(m+2)^{p}+(1+2 m)^{p}+6(1+m)^{p}+\frac{3}{2} m^{p}<1$ for all $m \geq m_{0}$.

Therefore, we obtain that

$$
\begin{aligned}
& \varepsilon^{*}(x):=\sum_{n=0}^{\infty} \Lambda^{n} \varepsilon(x)=\frac{1}{4} c\left((1+m)^{p}+m^{p}\right)\|x\|^{p} \sum_{n=0}^{\infty} \alpha_{m}{ }^{n} \\
& =\frac{c\left((1+m)^{p}+m^{p}\right)\|x\|^{p}}{4\left(1-\alpha_{m}\right)}<\infty, \forall x \in X \backslash\{0\} ; \quad \forall m \geq m_{0} .
\end{aligned}
$$

Thus according to Theorem 1.3, for each $m \geq m_{0}$ there exists a unique solution $F_{m}: X \backslash\{0\} \rightarrow Y$ of the equation

$$
\begin{aligned}
& F_{m}(x) \\
& =\frac{1}{4} F_{m}((3 m+1) x)+\frac{1}{4} F_{m}((m+2) x)-F_{m}((1+2 m) x)-6 F_{m}((1+m) x) \\
& +\frac{3}{2} F_{m}(m x) \forall x \in X \backslash\{0\}
\end{aligned}
$$

such that

$$
\left\|f(x)-F_{m}(x)\right\| \leq \varepsilon_{m}^{*}(x),, \quad x \in X \backslash\{0\}, \quad m \geq m_{0} .
$$


Moreover $F_{m}(x)=\lim _{n \rightarrow \infty} T_{m}^{n}(x), x \in X \backslash\{0\}$.

To prove that $F_{m}(x)$ satisfies (1.4) on $X \backslash\{0\}$, observe that

$$
\begin{aligned}
& \left\|\mathcal{T}^{n} f(x)-\left[\frac{1}{4} \mathcal{T}^{n} f(y)+\frac{1}{4} \mathcal{T}^{n} f(x+y)-\mathcal{T}^{n} f(x-y)-6 \mathcal{T}^{n} f(2 x+y)+\frac{3}{2} \mathcal{T}^{n} f(2 x-y)\right]\right\| \\
& \leq \frac{1}{4} c\left(\|x\|^{p}+\|y\|^{p}\right) \alpha_{m}^{n} .
\end{aligned}
$$

for all $x, y \in X \backslash\{0\}$ and $n \in \mathbf{N}$ such that $x+y \neq 0, x-y \neq 0,2 x+y \neq$ $0,2 x-y \neq 0$.

Indeed, if $n=0$, then (2.8) is simply (2.6). So, fix $n \in \mathbf{N}$ and suppose that (2.8) holds for $n$. Then

$$
\begin{aligned}
& \| T^{n+1} f(x)-\left[\frac{1}{4} T^{n+1} f(2 x+y)+\frac{1}{4} T^{n+1} f(2 x-y)-T^{n+1} f(x+y)\right. \\
& \left.-6 T^{n+1} f(x-y)+\frac{3}{2} T^{n+1} f(y)\right] \| \\
& =\| \frac{1}{4} T^{n} f((3 m+1)(x))+\frac{1}{4} T^{n} f((m+2)(x))-T^{n} f((1+2 m)(x)) \\
& -6 T^{n} f((1+m)(x))+\frac{3}{2} T^{n} f(m(x))-\left[\frac { 1 } { 4 } \left[\frac{1}{4} T^{n} f((3 m+1)(2 x+y))\right.\right. \\
& +\frac{1}{4} T^{n} f((m+2)(2 x+y))-T^{n} f((1+2 m)(2 x+y)) \\
& \left.-6 T^{n} f((1+m)(2 x+y))+\frac{3}{2} T^{n} f(m(2 x+y))\right]+\frac{1}{4}\left[\frac{1}{4} T^{n} f((3 m+1)(2 x-y))\right. \\
& +\frac{1}{4} T^{n} f((m+2)(2 x-y))-T^{n} f((1+2 m)(2 x-y)) \\
& \left.-6 T^{n} f((1+m)(2 x-y))+\frac{3}{2} T^{n} f(m(2 x-y))\right]-\left[\frac{1}{4} T^{n} f((3 m+1)(x+y))+\right. \\
& \frac{1}{4} T^{n} f((m+2)(x+y))-T^{n} f((1+2 m)(x+y) \\
& \left.-6 T^{n} f((1+m)(x+y))+\frac{3}{2} T^{n} f(m(x+y))\right] \\
& -6\left[\frac{1}{4} T^{n} f((3 m+1)(x-y))+\frac{1}{4} T^{n} f((m+2)(x-y))\right. \\
& \left.-T^{n} f((1+2 m)(x-y))-6 T^{n} f((1+m)(x-y))+\frac{3}{2} T^{n} f(m(x-y))\right] \\
& +\frac{3}{2}\left[\frac{1}{4} T^{n} f((3 m+1) y)+\frac{1}{4} T^{n} f((m+2)(y))\right. \\
& \left.\left.-T^{n} f((1+2 m)(y))-6 T^{n} f((1+m)(y))+\frac{3}{2} T^{n} f(m(y))\right]\right] .
\end{aligned}
$$


So

$$
\begin{aligned}
& \| T^{n+1} f(x)-\left[\frac{1}{4} T^{n+1} f(2 x+y)+\frac{1}{4} T^{n+1} f(2 x-y)-T^{n+1} f(x+y)\right. \\
& \left.-6 T^{n+1} f(x-y)+\frac{3}{2} T^{n+1} f(y)\right] \| \\
& =\| \frac{1}{4}\left[T^{n} f((3 m+1) x)-\left[\frac{1}{4} T^{n} f((3 m+1)(2 x+y))+\frac{1}{4} T^{n} f((3 m+1)(2 x-y))\right.\right. \\
& \left.\left.-T^{n} f((3 m+1)(x+y))-6 T^{n} f((3 m+1)(x-y))+\frac{3}{2} T^{n} f(m(y))\right]\right] \\
& +\frac{1}{4}\left[T^{n} f((m+2) x)-\left[\frac{1}{4} T^{n} f((m+2)(2 x+y))+\frac{1}{4} T^{n} f((m+2)(2 x-y))\right.\right. \\
& \left.\left.-T^{n} f((m+2)(x+y))-6 T^{n} f((m+2)(x-y))+\frac{3}{2} T^{n} f((m+2)(y))\right]\right] \\
& -\left[T^{n} f((1+2 m) x)-\left[\frac{1}{4} T^{n} f((1+2 m)(2 x+y))+\frac{1}{4} T^{n} f((1+2 m)(2 x-y))\right.\right. \\
& \left.\left.-T^{n} f((m+2)(x+y))-6 T^{n} f((1+2 m)(x-y))+\frac{3}{2} T^{n} f((1+2 m)(y))\right]\right] \\
& -6\left[T^{n} f((m+1) x)-\left[\frac{1}{4} T^{n} f((m+1)(2 x+y))+\frac{1}{4} T^{n} f((m+1)(2 x-y))\right.\right. \\
& \left.\left.-{ }^{n} f((m+1)(x+y))-6 T^{n} f((m+1)(x-y))+\frac{3}{2} T^{n} f((m+1)(y))\right]\right] \\
& +\frac{3}{2}\left[T^{n} f(m x)-\left[\frac{1}{4} T^{n} f m(2 x+y)+\frac{1}{4} T^{n} f(m(2 x-y))-T^{n} f(m(x+y))\right.\right. \\
& \left.\left.-6 T^{n} f(m(x-y))+\frac{3}{2} T^{n} f(m y)\right]\right] \| \\
& \leq \frac{1}{4}\left(\frac{1}{4} c\left(\|(3 m+1) x\|^{p}+\|(3 m+1) y\|^{p}\right) \alpha_{m}^{n}+\frac{1}{4} c\left(\|(m+) x\|^{p}\right.\right. \\
& \left.+\|(m+2) y\|^{p}\right) \alpha_{m}^{n}+c\left(\|(1+2 m) x\|^{p}+\|(1+2 m) y\|^{p}\right) \alpha_{m}^{n}+6 c\left(\|(1+m) x\|^{p}\right. \\
& \left.\left.+\|(1+m) y\|^{p}\right) \alpha_{m}^{n}+\frac{3}{2} c\left(\|m x\|^{p}+\|m y\|^{p}\right) \alpha_{m}^{n}\right) \\
& =\frac{1}{4} c\left(\left.\left.|| x\right|^{p}|+| y\right|^{p}\right)\left(\frac{1}{4}(3 m+1)^{p}+\frac{1}{4}(m+2)^{p}+(1+2 m)^{p}\right. \\
& \left.-6(1+m)^{p}+\frac{3}{2} m^{p}\right) \alpha_{m}^{n} \\
& =\frac{1}{4} c\left(\|x\|^{p}+\|y\|^{p}\right) \alpha_{m}^{n+1}
\end{aligned}
$$

for all $x, y \in X \backslash\{0\}$ such that $x+y \neq 0, x-y \neq 0,2 x+y \neq 0,2 x-y \neq 0$.

Thus, by induction, we have shown that (2.8) holds for all $x, y \in X \backslash\{0\}$ 
and for all $n \in \mathbf{N}$.

Letting $n \rightarrow \infty$ in (2.8), we obtain

$F_{m}(x)=\frac{1}{4} F_{m}(y)-\frac{1}{6} F_{m}(x+y)-\frac{1}{6} F_{m}(x-y)+\frac{1}{24} F_{m}(2 x+y)+\frac{1}{24} F_{m}(2 x-y)$

for all $x, y \in X \backslash\{0\}$ such that $x+y \neq 0, x-y \neq 0,2 x+y \neq 0,2 x-y \neq 0$. So, we find a sequence $\left(F_{m}\right)_{m \geq m_{0}}$ satisfies (1.4) on $X \backslash\{0\}$ such that

$$
\left\|f(x)-F_{m}(x)\right\| \leq \frac{c m^{p}}{4\left(1-\alpha_{m}\right)}\|x\|^{p}, \forall x \in X-\{0\}, \forall m \geq m_{0} .
$$

It follows, with $m \rightarrow \infty$, that $f$ is a solution of (1.4) on $X \backslash\{0\}$.

Corollary 2.2.1. Let $X \backslash\{0\}$ be a normed space and $Y$ be a Banach space. Let $H: X \backslash\{0\}^{2} \rightarrow Y$ be a mapping such that $H\left(x_{0}, y_{0}\right) \neq 0$ for some $x_{0}, y_{0} \in X \backslash\{0\}$ and

$$
\|H(x, y)\| \leq c\left(\|x\|^{p}+\|y+\|^{p}\right),
$$

for all $x, y \in X \backslash\{0\}$ such that $x+y \neq 0, x-y \neq 0,2 x+y \neq 0,2 x-y \neq 0$, where $c \geq 0$ and $p \in \mathbf{R}$. Assume that the numbers $p$ satisfy the following condition: $p<0$. Then the functional equation

$H(2 x+y)+H(2 x-y)=H(x, y)+4(H(x+y)+H(x-y))+24 H(x)-6 H(y)$,

$\forall x, y \in X \backslash\{0\}$ such that $x+y \neq 0, x-y \neq 0,2 x+y \neq 0,2 x-y \neq 0$, has no solution in the class of functions $H: X \rightarrow Y$.

Proof. Suppose that $H: X \rightarrow Y$ is a solution to (2.10). Then (2.6) holds, and consequently, according to the above theorem, $H$ is quartic function equation on $X \backslash\{0\}$, which means that $H\left(x_{0}, y_{0}\right)=0$. This is a contradiction. 


\section{References}

[1] T. Aoki, On the stability of the linear transformation in Banach spaces, J. Math. Soc. Japan, 2, pp. 64-66, (1950).

[2] C. Baak, Cauchy-Rassias stability of Cauchy-Jensen additive mappings in Banach spaces, Acta Mathematica Sinica, English Series, Vol.22, No.6, pp. 1789-1796, (2006).

[3] A. Bahyrycz, M. Piszczek, Hyperstability of the Jensen functional equation, Acta Math. Hungar. 142 (2), pp. 353-365, (2014).

[4] D. G. Bourgin, Approximately isometric and multiplicative transformations on continuous function rings, Duke Math. J., 16, pp. 385-397, (1949).

[5] D. G. Bourgin, Classes of transformations and bordering transformations, Bull. Amer. Math. Soc., 57, pp. 223-237, (1951).

[6] J. Brzdek, K. Cienplinski , Hyperstability and superstability. Abstract and Applied Analysis. Article ID 101756 13pp., (2013).

[7] J. Brzdek, J. Chudziak, Z. Páles, A fixed point approach to stability of functional equations, Nonlinear Anal., Vol. 74, No. 17, pp. 6728-6732, (2011).

[8] J. Brzdek, Remarks on hyperstability of the the Cauchy equation, Aequations Mathematicae, 86, pp. 255-267, (2013).

[9] J. Brzdek, W. Fechner, M. S. Moslehian, J. Sikorska, Recent developments of the conditional stability of the homomorphism equation, Banach J. Math. Anal. 9, No. 3, pp. 278-326, (2015).

[10] J. Brzdek, Hyperstability of the Cauchy equation on restricted domains, Acta Math. Hungarica, 141 (1-2), pp. 58-67, (2013).

[11] J. Brzdek, A hyperstability result for the Cauchy equation. Bulletin of the Australian Mathematical Society 89, pp. 33-40, (2014).

[12] I. I. EL-Fassi, , S. Kabbaj, On the hyperstability of a Cauchy-Jensen type functional equation in Banach spaces. Proyecciones (Antofagasta), 34 (4), pp. 359-375, (2015). 
[13] Z. Gajda, On stability of additive mappings, Int. J. Math. Math. Sci. 14, pp. 431-434, (1991).

[14] E. Gselmann, Hyperstability of a functional equation, Acta Mathematica Hungarica, vol. 124, No. 1-2, pp. 179-188, (2009).

[15] D. H. Hyers, On the stability of the linear functional equation, Proc. Nat. Acad. Sci., U.S.A., 27, pp. 222-224, (1941).

[16] S. H. Lee, Im and I. S. Shwang, Quartic functional equations, J. Math. Anal. Appl 307, pp. 387-394, (2005).

[17] G. Maksa, Z. Páles, Hyperstability of a class of linear functional equations, Acta Math., vol. 17, no. 2, pp. 107-112, (2001).

[18] M. Piszczek, Remark on hyperstability of the general linear equation, Aequations Mathematicae, (2013).

[19] Th. M. Rassias, On the stability of the linear mapping in Banach spaces, Proc. Amer. Math. Soc., 72, pp. 297-300, (1978).

[20] Th. M. Rassias, On a modified HyersUlam sequence, J. Math. Anal. Appl. 158, pp. 106-113, (1991).

[21] Th. M. Rassias and P. Semrl, On the behavior of mappings which do not satisfy Hyers-Ulam stability, Proc. Amer. Math. Soc. 114, pp. 989-993, (1992).

[22] J. M. Rassias, solution of the Ulam stability problem for quartic mapping, Glasmik, Matematicki 34 (54), pp. 243-252, (1999).

[23] S. M. Ulam, Problems in Modern Mathematics, Chapter IV, Science Editions, Wiley, New York, (1960).

\section{Nordine Bounader}

Department of Mathematics,

Faculty of Sciences,

University of Ibn Tofail,

Kenitra,

Morocco

e-mail : n.bounader@live.fr 